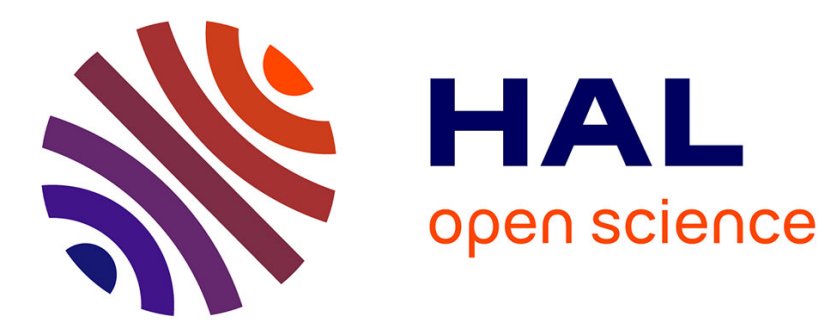

\title{
De Bruijn isomorphisms and free space optical networks
}

\author{
David Coudert, Afonso Ferreira, Stéphane Pérennes
}

\section{To cite this version:}

David Coudert, Afonso Ferreira, Stéphane Pérennes. De Bruijn isomorphisms and free space optical networks. 14th International Parallel and Distributed Processing Symposium (IPDPS 2000), May 2000, Cancun, Mexico. pp.769 - 774, 10.1109/IPDPS.2000.846063 . inria-00429190

\section{HAL Id: inria-00429190 https://hal.inria.fr/inria-00429190}

Submitted on 1 Nov 2009

HAL is a multi-disciplinary open access archive for the deposit and dissemination of scientific research documents, whether they are published or not. The documents may come from teaching and research institutions in France or abroad, or from public or private research centers.
L'archive ouverte pluridisciplinaire HAL, est destinée au dépôt et à la diffusion de documents scientifiques de niveau recherche, publiés ou non, émanant des établissements d'enseignement et de recherche français ou étrangers, des laboratoires publics ou privés. 


\title{
De Bruijn Isomorphisms and Free Space Optical Networks
}

\author{
D. Coudert \\ A. Ferreira \\ S. Perennes \\ Project SLOOP - CNRS-I3S-INRIA \\ 2004, route des Lucioles - BP 93 \\ F-06902 Sophia-Antipolis \\ France \\ \{David.Coudert, Afonso.Ferreira, Stephane.Perennes\}@ sophia.inria.fr
}

\begin{abstract}
The de Bruijn digraph $B(d, D)$ is usually defined by words of size $D$ on an alphabet of cardinality d, through a cyclic left shift permutation on the words, after which the rightmost symbol is changed. In this paper, we show that any digraph defined on words and alphabets of the same size, through an arbitrary permutation on the alphabet and an arbitrary permutation on the word indices, is isomorphic to the de Bruijn, provided that this latter permutation is cyclic. As an application, we recall that it is known that the Optical Transpose Interconnection System from UCSD can implement the de Bruijn interconnections for $n$ nodes, for a fixed d, with $O(n)$ lenses. We show here how to improve this hardware requirement to $\Theta(\sqrt{n})$.
\end{abstract}

Keywords: Distributed systems, Optical networks, Free space of optical interconnections, Optical Transpose Interconnection System, de Bruijn, Graph isomorphisms, Digraphs.

\section{Introduction}

Many results exist in the literature describing technological and theoretical advances in optical network topologies and design. However, an essential effort has yet to be done in linking those results together. In this paper, we propose a step in this direction, by giving optimal optical layouts of several de Bruijn-like topologies, using existing optical technologies, namely the Optical Transpose Interconnecting System (OTIS) architecture, from the Optoelectronic Computing Group of the UCSD $[6,25]$. These results are obtained using graph theoretical properties that we prove in this paper. In fact, our optical layouts are particular cases of more general graph isomorphisms that we develop here.
Our motivation stems from the fact that it has been shown that the break-even line length where optical communication lines become more effective than their electrical counterparts is less than $1 \mathrm{~cm}$, in terms of speed and power consumption [16]. Therefore, the use of optical interconnections on-board is nowadays justified, and some studies even suggest that on-chip optical interconnects will soon be cost-effective [33]. Moreover, the emergence of cutting-edge technologies as Vertical Cavity Surface-Emitting Lasers (VCSELs) [15,31], high sensibility optical transimpedance receivers [5], beam splitters [17, 18], micro-lenses [26], and holograms [7], makes possible the fabrication of complex optical communication networks.

The OTIS architecture is a simple scalable means of implementing very dense one-to-one interconnections between processors in a free space of optical interconnections. In a nutshell, it consists of two lenslet arrays allowing a large number of optical interconnections from a set of transmitters to a set of receivers. The OTIS architecture is used by the 3D OptoElectronic Stacked Processors consortium to connect a 16-by-16 switch (For more details, see http://soliton.ucsd.edu/3doesp/public/).

It has been shown in [34] that the OTIS architecture can be used to build all-optical complete networks, based on a symmetric complete digraph with loops $\left(K_{n}^{*}\right)$. The OTIS architecture and recent progress made in optoeletronics [24] allow the layout of a 64 processors complete digraph, each processor having 64 transceivers (corresponding to the 64 arcs of one vertex). It has also been shown in [14] how to realize the single-hop multiOPS POPS network [10], and the multi-hop multi-OPS stack-Kautz network [13] with the OTIS architecture. However, these layouts scale badly, since they require a large number of transceivers per processor, as well as a large number of optical lenses. Consequently, an important research direction is to study the set of network 
topologies for which there are efficient layouts with the OTIS architecture.

In our work, we focus on de Bruijn-like digraphs, since the de Bruijn [1] is a well known digraph which has been extensively studied in graph theory, with applications in parallel and distributed computing. A de Bruijn digraph $B(d, D)$ is an alphabet digraph of constant degree $d$ and diameter $D$ having for vertices the $d^{D}$ words of length $D$ on the alphabet $Z_{d}=$ $\{0,1, \ldots, d-1\}$, such that a vertex $x=x_{D-1} \ldots x_{1} x_{0}$, $x_{i} \in Z_{d}$, is adjacent to the set of vertices $\Gamma^{+}(x)=$ $\left\{x_{D-2} \ldots x_{1} x_{0} \beta, \beta \in Z_{d}\right\}[1]$. Many results exist on routing [19], broadcasting [28], gossiping [3] and embedding [9] on this digraph as well as on parallel algorithms for it $[12,30]$. Furthermore, it has been used to build various multi-stage interconnection networks like Butterfly [30], Shufflenet [27] and GEMNET [27] and also to build fully scalable networks of any size [22] and very large bus networks [2]. More interestingly, the Galileo space probe of the NASA uses a de Bruijn network to implement a signal decoder [11].

From a theoretical point of view, we show in this paper that there are many ways to build digraphs isomorphic to the de Bruijn. For this, we start showing, in Proposition 3.2, that the alphabet digraph $B_{\pi}(d, D)$, built using a permutation $\pi$ on $Z_{d}$ and the adjacency relation $\Gamma_{B_{\pi}(d, D)}^{+}(x)=$ $\left\{\pi\left(x_{D-2}\right) \ldots \pi\left(x_{1}\right) \pi\left(x_{0}\right) \pi(\beta), \beta \in Z_{d}\right\}$, is isomorphic to $B(d, D)$. As a corollary, it follows the isomorphism between the de Bruijn digraph and the Imase and Itoh digraph [20], a digraph issued from the minimumdiameter maximum-size problem and which is usually said to be a generalization of the Kautz digraph [23]. Then, we show, in Proposition 3.9, that there is a permutation $f$ on $Z_{D}$ which defines an alphabet digraph, isomorphic to $B(d, D)$, if and only if $f$ is cyclic. Notice that the permutation $\pi$ is applied on the alphabet and $f$ is applied on the word indices (see Section 3.2). Finally, we show that the permutations $\pi$ and $f$ may be applied together, which characterizes a class of digraphs which are isomorphic to the de Bruijn.

As an application of these results, we recall that it has been proved that the Imase and Itoh digraph, $I I(d, n)$, with $n$ nodes of degree $d$, can be implemented by the OTIS architecture [14]. Our result on the isomorphism of $I I(d, n)$ and $B(d, D)$ yields that OTIS can also implement the de Bruijn. Unfortunately, for a fixed degree $d$, such a layout uses $O(n)$ lenses to interconnect $n$ processors, which is not very efficient. Our subsequent results help us to show how OTIS can implement the de Bruijn with only $\Theta(\sqrt{n})$ lenses, which is not only optimal, but also very efficient for practical values, as it will be discussed later.
This paper is organized as follows. In Section 2, we give the graph theoretical machinery which we will need in the following. In particular, we remind the definition of the de Bruijn digraph and its generalization, the Reddy-Raghavan-Kuhl digraph [29]. We also recall the definition of the Imase and Itoh digraph, known to be a Kautz digraph generalization. Then, in Section 3, we show that there are many isomorphisms of the de Bruijn digraph, using first a permutation $\pi$ on the alphabet $Z_{d}$ and then a permutation $f$ on the indices $Z_{D}$. We hence obtain $d !(D-1)$ ! such isomorphic digraphs. The application to optical networks is given in Section 4, where we present OTIS layouts of de Bruijn-like digraphs and we show how to obtain such layouts which minimize the number of lenses used. We close the paper with some concluding remarks and directions for further research.

\section{Preliminaries}

\subsection{Notations}

As usually, $Z_{n}$ will denote the ring of integers modulo $n . Z_{n}=\{0,1,2, \ldots, n-1\} . Z_{n}^{k}$ will denotes the $k$ dimensional vector space on $Z_{n}$ and $e_{0}, e_{1}, \ldots, e_{n-1}$ the canonical base. Addition of elements of $Z_{n}$ will always be performed in $Z_{n}$ (i.e modulo $n$ ).

For a permutation $f$ and an integer $i$, we define $f^{i}$ inductively by taking $f^{0}$ as the identity permutation and $f^{i+1}=f \circ f^{i}$.

The next permutation will be used frequently:

Definition 2.1 We will denote $\mathcal{C}$ the complement permutation of $Z_{n}$ defined by $\mathcal{C}(u)=n-u-1$. As usually $\mathcal{C}(u)$ will be also denoted $\bar{u}$.

\subsection{Classical Digraphs}

Given a digraph $G=(V, E)$, and $x \in V$ we will denote $\Gamma_{G}^{+}(x)$ the set of the out-neighbors of $x$, i.e. $\{y \in$ $V,(x, y) \in E\}$. When the digraph $G$ is clear from the context we simply denote $\Gamma^{+}(x)$.

Definition 2.2 [1] The de Bruijn digraph, $B(d, D)$, of degree $d$ and diameter $D$ is defined as a digraph on the set of vertices made of words of length $D$ on $Z_{d}$, and such that for $x=x_{D-1} x_{D-2} \cdots x_{1} x_{0}, \Gamma^{+}(x)=$ $\left\{x_{D-2} \cdots x_{1} x_{0} \beta, \beta \in Z_{d}\right\}$.

Definition 2.3 The conjunction $G_{1} \otimes G_{2}$ of two digraphs $G_{1}=\left(V_{1}, E_{1}\right)$ and $G_{2}=\left(V_{2}, E_{2}\right)$ is the digraph with vertex-set $V_{1} \times V_{2}$, and where there is an arc from $\left(u_{1}, u_{2}\right)$ to $\left(v_{1}, v_{2}\right)$ if and only if there is an both an arc from $u_{1}$ to $v_{1}$ in $G_{1}$ and an arc from $u_{2}$ to $v_{2}$ in $G_{2}$. 
Alternatively we have $\Gamma_{G_{1} \otimes G_{2}}^{+}\left(\left(u_{1}, u_{2}\right)\right)=\Gamma_{G_{1}}^{+}\left(u_{1}\right) \times$ $\Gamma_{G_{2}}^{+}\left(u_{2}\right)$.

Remark 2.4 $B(d, k) \otimes B\left(d^{\prime}, k\right)=B\left(d d^{\prime}, k\right)$; one can see [4,32] for more details about conjunction and de Bruijn digraphs and line digraphs.

Definition 2.5 [29] The Reddy-Raghavan-Kuhl digraph, $R R K(d, n)$, of degree $d$ with $n$ nodes, is the digraph with vertex set $Z_{n}$ and such that for $u \in Z_{n}$ : $\Gamma^{+}(u)=\{d u+\alpha, 0 \leq \alpha<d\}$.

Remark 2.6 The digraphs $R R K\left(d, d^{D}\right)$ and $B(d, D)$ are isomorphic (see [29]). To see it, we associate to $a$ vertex $x=x_{D-1} x_{D-2} \cdots x_{1} x_{0}$ of $B(d, D)$ a number $u \in Z_{n}$ with $u=\sum_{i=0}^{D-1} x_{i} d^{i}$; then the out-neighbors of $x$ in $B(d, D)$ are associated to numbers of the form du+ $\alpha, 0 \leq \alpha<d$. Note also that this congruent definition is sometimes considered as the standard one for the de Bruijn digraph.

Definition 2.7 [23] The Kautz digraph, $K(d, D)$, of degree $d$ and diameter $D$ is defined as a digraph on the set of vertices made of words of length $D$ on the alphabet $Z_{d+1}, x=x_{D-1} \cdots x_{1} x_{0}$, with $x_{i} \in Z_{d+1}$ and $x_{i} \neq$ $x_{i+1} . \Gamma^{+}(x)=\left\{x_{D-2} \cdots x_{1} x_{0} \beta, \beta \neq x_{0}, \beta \in Z_{d+1}\right\}$.

Definition 2.8 [20] The Imase and Itoh digraph, $I I(d, n)$, of degree $d$ with $n$ nodes, is the digraph with vertex set $Z_{n}$ and such that for $u \in Z_{n}: \Gamma^{+}(u)=$ $\{-d u-\alpha, 1 \leq \alpha \leq d\}$.

It has been shown in [21] that $I I\left(d, d^{D-1}(d+1)\right)$ is isomorphic to $K(d, D)$.

\section{Alternative definition of $B(d, D)$ as a di- graph on alphabet}

In this section we define generalizations of de Bruijn digraph. In order to obtain the out-neighbors of a vertex labeled by some word of length $D$ over $Z_{d}$ we proceede as follows:

- instead of performing a left-shift on the word letters we permute the letter positions in some arbitrary way;

- then each letter is replaced by another one according to some fixed permutation of the alphabet $Z_{d}$;

- finally the letter of a given fixed position is replaced by an arbitrary letter of $Z_{d}$.

\subsection{Permutation on the alphabet}

We start by considering only the case of a permutation on the alphabet. We will show that there is an isomorphism between $B(d, D)$ and digraphs that we call $B_{\pi}(d, D)$, i.e., which are similar to $B(d, D)$ up to a permutation on the alphabet $Z_{d}$. Then we will prove that $I I\left(d, d^{D}\right)$ and $B(d, D)$ are isomorphic, which, according to the Remark 2.6 will imply that $I I\left(d, d^{D}\right), B(d, D), R R K\left(d, d^{D}\right)$ are isomorphic.

Definition 3.1 Given a permutation $\pi$ of $Z_{d}, B_{\pi}(d, D)$ is defined as the digraph on the set of vertices made of words of length $D$ on $Z_{d}$, such that for each vertex $x=x_{D-1} x_{D-2} \cdots x_{1} x_{0}, x_{i} \in Z_{d}, \Gamma^{+}(x)=$ $\left\{\pi\left(x_{D-2}\right) \cdots \pi\left(x_{0}\right) \beta, \beta \in Z_{d}\right\}$.

Proposition 3.2 $B(d, D)$ and $B_{\pi}(d, D)$ are isomorphic.

Proof: We just have to define an isomorphism $W$ from $B_{\pi}(d, D)$ to $B(d, D) ; W$ is the following one to one mapping from the words of length $D$ on the alphabet $Z_{d}$ onto itself:

$$
\begin{aligned}
& W\left(x_{D-1} x_{D-2} \ldots x_{1} x_{0}\right) \\
& =\pi^{0}\left(x_{D-1}\right) \pi^{1}\left(x_{D-2}\right) \ldots \pi^{D-2}\left(x_{1}\right) \pi^{D-1}\left(x_{0}\right)
\end{aligned}
$$

Given a vertex $x$ of $B_{\pi}(d, D)$ we have:

$$
\begin{aligned}
& W^{-1} \Gamma_{B(d, D)}^{+}\left(W\left(x_{D-1} x_{D-2} \ldots x_{0}\right)\right) \\
& =W \Gamma_{B(d, D)}^{+}\left(\pi^{0}\left(x_{D-1}\right) \pi^{1}\left(x_{D-2}\right) \ldots \pi^{D-1}\left(x_{0}\right)\right) \\
& =W^{-1}\left(\pi^{1}\left(x_{D-2}\right) \ldots \pi^{D-2} x_{1} \pi^{D-1}\left(x_{0}\right) \beta\right), \beta \in Z_{d} \\
& =\pi\left(x_{D-2}\right) \ldots \pi\left(x_{0}\right) \pi(\beta) \\
& =\pi\left(x_{D-2}\right) \ldots \pi\left(x_{0}\right) \alpha, \alpha \in Z_{d} .
\end{aligned}
$$

As the last line corresponds to the adjacency relation in $B_{\pi}(d, D)$ we have defined the required isomorphism.

Notice that more generally, the digraph of degree $d$, diameter $D$ and $n=d^{D}$ nodes, such that $\Gamma^{+}\left(x_{D-1} x_{D-2} \ldots x_{1} x_{0}\right)=$ $\left\{\pi_{0}\left(x_{D-2}\right) \pi_{1}\left(x_{D-3}\right) \ldots \pi_{D-2}\left(x_{0}\right) \pi_{D-1}(\alpha), \quad \alpha \quad \in\right.$ $\left.Z_{d}\right\}$, where $\pi_{i}, i \in Z_{D}$, is a permutation on $Z_{d}$, is isomorphic to $B(d, D)$.

Proposition 3.3 The digraphs $B(d, D)$ and $I I\left(d, d^{D}\right)$ are isomorphic.

Proof: Let $\bar{B}(d, D)$ denote $B_{\mathcal{C}}(d, D)$, where $\mathcal{C}$ is the complement permutation over $Z_{d}$. As in the case of $B(d, D), \bar{B}(d, D)$ can be alternatively defined as a digraph with vertex set $Z_{n}$ (see Remark 2.6) in which for $u \in Z_{n}, \Gamma^{+}(u)=\{-(d u+\beta)-1,0 \leq \beta<d\}$, that 
is $\Gamma^{+}(u)=\{-d u-\alpha, 1 \leq \alpha \leq d\}$, This is the definition of $I I\left(d, d^{D}\right)$. So $B_{\mathcal{C}}(d, D)$ and $I I\left(d, d^{D}\right)$ are isomorphic. From proposition 3.2 these digraphs are hence isomorphic to $B(d, D)$.

By Remark 2.6, we obtain:

Corollary 3.4 The digraphs $B(d, D), \quad R R K\left(d, d^{D}\right)$ and $I I\left(d, d^{D}\right)$ are isomorphic.

To illustrate our purpose, Figure 1 shows the digraph $B(2,3)$ which is isomorphic to $R R K(2,8)$ of Figure 2 and to $I I(2,8)$ represented on Figure 3.

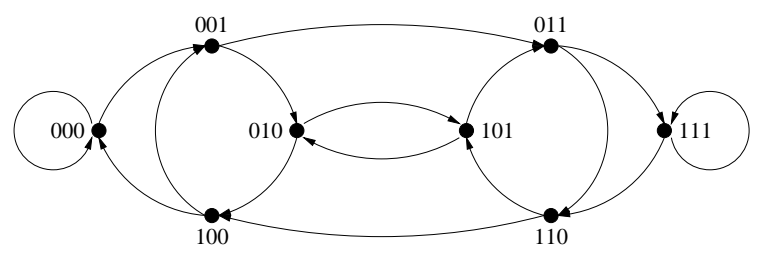

Figure 1. de Bruijn of degree 2, diameter 3 and 8 nodes.

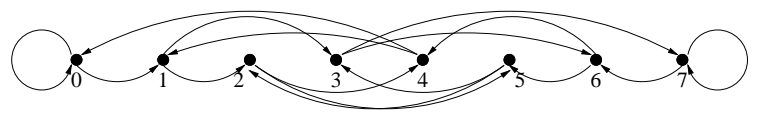

Figure 2. RRK digraph of degree 2, diameter 3 and 8 nodes.

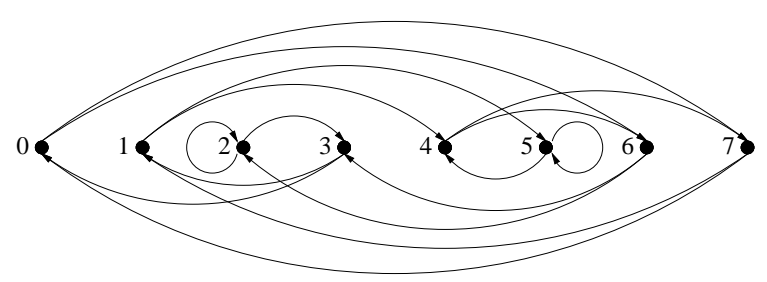

Figure 3. II digraph of degree 2, diameter 3 and 8 nodes.

\subsection{Permutation on the indices, digraphs $A(f, \pi, s)$}

Now we consider more general digraphs than $B_{\pi}(d, D)$ for which the left shift is replaced by any permutation of $Z_{D}$. First we need some definitions.
Definition 3.5 To a permutation $f$ of $Z_{D}$, we associate the linear map $\vec{f}$ of $Z_{d}^{D}$ defined on the basis by $\vec{f}\left(e_{i}\right)=e_{f(i)}$ (this linear map is associated with a permutation matrix). Note that $\overrightarrow{f g}=\vec{f} \vec{g}$.

Definition 3.6 Any permutation $\pi$ on $Z_{d}$, is extended naturally to $Z_{d}^{D}$ by setting for $x=x_{D-1} x_{D-2} \cdots x_{1} x_{0}$ : $\pi(x)=\pi\left(x_{D-1}\right) \pi\left(x_{D-2}\right) \cdots \pi\left(x_{1}\right) \pi\left(x_{0}\right)$.

Definition 3.7 Let $d, D \in N$, given a permutation $f$ on $Z_{D}$, a permutation $\pi$ of $Z_{d}$ and a position $j \in Z_{D}$, we define the digraph $G=A(f, \pi, j)$ by:

$$
\begin{aligned}
V(G) & =Z_{d}^{D} \\
\Gamma_{G}^{+}(x) & =\pi(f(x))+Z_{d} \cdot e_{j}
\end{aligned}
$$

$A(f, \pi, j)$ has degree $d$ and we will say that it has dimension D.

Remark 3.8 Note that vertices of $B(d, D)$ can be considered as elements of $Z_{d}^{D}$ (by associating $x_{D-1} \cdots x_{1} x_{0}$ to $\left.\sum x_{i} e_{i}\right)$. Let $\sigma$ be the permutation of $Z_{D}$ defined by $\sigma: i \quad r \quad i+1$ $\bmod D$, and $I d$ be the identity permutation on $Z_{d}$ then the de Bruijn digraph is exactly $A(\sigma, I d, 0)$. Indeed for $x=x_{D-1} x_{D-2} \cdots x_{1} x_{0}$, $\Gamma_{A(\sigma, I d, 0)}^{+}(x)=\left\{x_{D-2} \cdots x_{1} x_{0}\left(x_{D-1}+\gamma\right), \gamma \in Z_{d}\right\}$, that is also $\left\{x_{D-2} \cdots x_{1} x_{0} \beta, \beta \in Z_{d}\right\}$. Similarly, $B_{\pi}(d, D)$ and $A(\sigma, \pi, 0)$ are isomorphic.

Proposition 3.9 The graph $G=A(f, \pi, j)$ with degree $d$ and dimension $D$ is isomorphic to $B(d, D)$ if and only if $f$ is a cyclic permutation of $Z_{D}$. Otherwise $A(f, \pi, j)$ is not connected.

Proof: Note that since $f$ is a cyclic permutation it induces a unique orbit on $Z_{D}$. We associate to $f$ the unique permutation $g$ of $Z_{D}$, such that $\forall i \in Z_{D}, g(i)=$ $f^{i}(j)$. From definition we have

1. $g^{-1} f g(i)=g^{-1} f f^{i}(j)=g^{-1} f^{i+1}(j)=$ $g^{-1} g(i+1)=i+1$

2. $g^{-1}(j)=0$ (Hence $\left.\overrightarrow{g^{-1}}\left(e_{j}\right)=e_{0}\right)$

Let us consider the digraph isomorphism induced by $\vec{g}$. For $x \in Z_{d}^{D}$, we compute $\overrightarrow{g^{-1}} \Gamma_{G}^{+}(\vec{g}(x))$. From definition of $G$ we have $\Gamma_{G}^{+}(x)=\pi(\vec{f}(x))+$ $Z_{d} . e_{j}$, thus by linearity we obtain $\overrightarrow{g^{-1}} \Gamma_{G}^{+}(\vec{g}(x))=$ $\pi\left(\overrightarrow{g^{-1}}(\vec{f} \vec{g}(x))\right)+Z_{d} \overrightarrow{g^{-1}}\left(e_{j}\right)$. But from 1) $\overrightarrow{g^{-1} f g}=$ $\vec{\sigma}$ and from 2) that $\overrightarrow{g^{-1}}\left(e_{j}\right)=e_{0}$. Finally, $\vec{g}-1 \Gamma_{G}^{+}(g(x))=\pi(\vec{\sigma}(x))+Z_{d} \cdot e_{0}$. And this adjacency relation is the one of $B_{\pi}(d, D)$ which is isomorphic to $B(d, D)$. We omit the proof that $A(f, \pi, j)$ is connected only if $f$ is cyclic. 
Notice that since there are $(D-1)$ ! cyclic permutation functions $f$ on $Z_{D}$, Proposition 3.9 leads to $(D-1)$ ! alternative ways of defining $B(d, D)$. Furthermore, as Proposition 3.2 leads to $d$ ! other ways of defining $B(d, D)$, using permutations $\pi$ on $Z_{d}$, we finally obtain $d !(D-1)$ ! alternative ways of defining $B(d, D)$.

Remark 3.10 If $f$ is not cyclic, $A(f, \pi, s)$ is not connected. Moreover, it can be proved that each connected component of $A(f, \pi, s)$, is the conjunction of a de Bruijn digraph with a circuit.

\subsection{Examples}

For the sake of illustration of Proposition 3.9, the following examples show, first, an alphabet digraph $H=$ $A(f, I d, 2)$ which is isomorphic to $B(d, 6)$, and then an alphabet digraph $H=A(f, I d, 1)$ where the permutation $f$ is not cyclic and hence $H$ is not connected.

3.3.1. $H=A(f, I d, 2)$

Let $H$ be the digraph of degree $d$, dimension 6 and vertex set $Z_{d}^{6}$, defined below:

$$
\Gamma_{H}^{+}\left(x_{5} x_{4} x_{3} x_{2} x_{1} x_{0}\right)=x_{2} x_{1} x_{0} \beta x_{5} x_{4} \text { with } \beta \in Z_{d} .
$$

$H$ is indeed $A(f, I d, 2)$, where $f$ is the following permutation of $Z_{6}$ :

$$
f(i)= \begin{cases}i+3 & \text { if } i<3 \\ 2 & \text { if } i=3 \\ i+2 \bmod 6 & \text { otherwise. }\end{cases}
$$

Then, $\vec{f}$ is defined on $Z_{d}^{6}$ by:

$$
\vec{f}\left(x_{5} x_{4} x_{3} x_{2} x_{1} x_{0}\right)=x_{2} x_{1} x_{0} x_{3} x_{5} x_{4}
$$

The permutation $g$ associated with $f$, used in Proposition 3.9 (i.e., $g(i)=f^{i}(2)$ ), is such that $g(0)=$ $2, g(1)=5, g(2)=1, g(3)=4, g(4)=0, g(5)=3$, and $\vec{g}\left(x_{5} x_{4} x_{3} x_{2} x_{1} x_{0}\right)=x_{1} x_{3} x_{5} x_{0} x_{2} x_{4}$, and hence $\overrightarrow{g^{-1}}\left(x_{5} x_{4} x_{3} x_{2} x_{1} x_{0}\right)=x_{3} x_{0} x_{4} x_{1} x_{5} x_{2}$. See Figure 4 for an illustration.

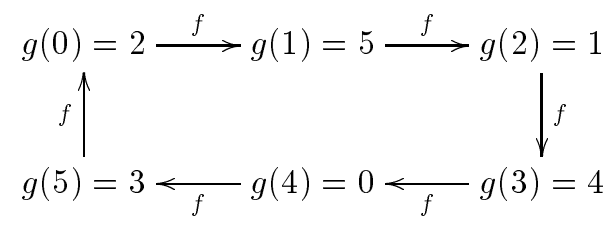

Figure 4. Illustration of the permutation $g$ defined as $g(i)=f^{i}(2)$.
Then,

$$
\begin{aligned}
\overrightarrow{g^{-1}} & \Gamma_{H}^{+} \vec{g}\left(x_{5} x_{4} x_{3} x_{2} x_{1} x_{0}\right) \\
& =\overrightarrow{g^{-1}} \Gamma_{H}^{+}\left(x_{1} x_{3} x_{5} x_{0} x_{2} x_{4}\right) \\
& =\overrightarrow{g^{-1}}\left(x_{0} x_{2} x_{4} \beta x_{1} x_{3}\right), \beta \in Z_{d} \\
& =x_{4} x_{3} x_{2} x_{1} x_{0} \beta \\
& =\Gamma_{B(d, 6)}^{+}\left(x_{5} x_{4} x_{3} x_{2} x_{1} x_{0}\right) .
\end{aligned}
$$

Therefore, $H$ is isomorphic to $B(d, 6)$.

$$
\text { 3.3.2. } H=A(f, I d, 1)
$$

Let $H$ be the digraph of degree $d$, dimension 3 , and vertex set $Z_{d}^{3}$, defined below:

$$
\Gamma_{H}^{+}\left(x_{2} x_{1} x_{0}\right)=x_{0} \beta x_{2}, \text { with } \beta \in Z_{d} .
$$

$H$ is indeed $A(f, I d, 1)$, where $f$ is the permutation of $Z_{3}$ such that $f(i)=\bar{i}=2-i$. Then, $\vec{f}$ is defined on $Z_{d}^{3}$ by $\vec{f}\left(x_{2} x_{1} x_{0}\right)=x_{0} x_{1} x_{2}$.

If we define the function $g$ associated to $f$ by $g(i)=$ $f^{i}(1)$, we get $g(0)=g(1)=g(2)=1$, hence $g$ is not a cyclic permutation of $Z_{3}$ and $H$ is not connected. Notice that the digraph $H$ can be alternatively defined by

$$
\Gamma_{H}^{+}\left(\left[x_{2} x_{0}\right],\left[x_{1}\right]\right)=\left\{\left[x_{0} x_{2}\right],[\beta]\right\}
$$

It is composed of $\frac{d^{2}-d}{2}$ digraphs $C_{2} \otimes B(d, 1)$ plus $d$ digraphs $C_{1} \otimes B(d, 1)$, as shown in Figure 5 for $d=2$.

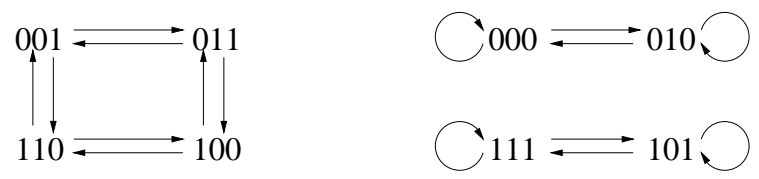

Figure 5. The digraph $H$ of example 3.3.2, when $d=2$

\section{OTIS layout for digraphs}

In this section, we first recall the definition of the OTIS architecture, and the known digraphs having a layout with OTIS. Then, we study the particular case of the OTIS layout of de Bruijn digraphs, and, using the theoretical results developed above, we show how to obtain optimal OTIS layouts for the de Bruijn.

\subsection{The OTIS architecture}

OTIS $(p, q)$ is a free-space optical system which allows one-to-one communications from $p$ groups of $q$ 
transmitters onto $q$ groups of $p$ receivers, using $p+q$ lenses. This architecture connects the transmitter $(i, j)$, $0 \leq i \leq p-1,0 \leq j \leq q-1$, to the receiver $(q-j-1, p-i-1)$. Optical interconnections in the OTIS architecture are realized with a pair of lenslet arrays [6] in a free space of optical interconnections as shown in Figure 6.

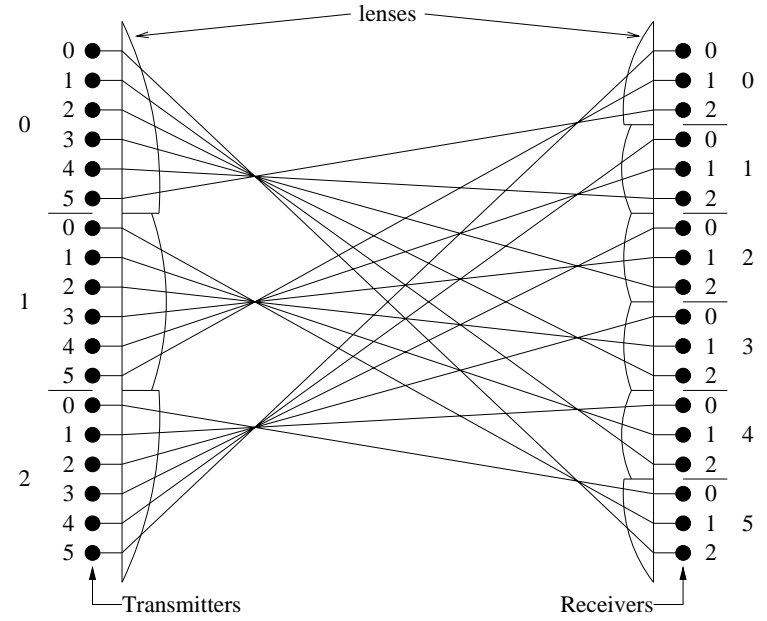

Figure 6. $O T I S(3,6)$.

\subsection{Known OTIS layouts}

Let $m=p q$. The OTIS $(p, q)$ architecture connects $m$ transmitter units of the processors to $m$ receiver units of the same set of processors. The interconnection network can be formally defined as follows. Given a degree $d$ such that $d$ divides $m$, let $H(p, q, d)$ be the $d$-regular digraph with $n=\frac{m}{d}$ vertices and vertex set $Z_{n}$, in which the $d$ transmitters $\left(\left\lfloor\frac{d u+\alpha}{q}\right\rfloor, d u+\alpha \bmod q\right), \alpha \in Z_{d}$, and the $d$ receivers $\left(\left\lfloor\frac{d u+\alpha}{p}\right\rfloor, d u+\alpha \bmod p\right), \alpha \in$ $Z_{d}$, belong to the node $u \in Z_{n}$ (i.e., a processing unit). There is a connection from the node $u$ to the node $v$ in $H(p, q, d)$ if one transmitter of $u$ is connected to one receiver of $v$. For instance, figure 7 represent the digraph $H(4,8,2)$.

Let $G=(V, E)$ be a digraph, with $|V|=n$ nodes of constant degree $d$ and $|E|=m=d n$ arcs. We say that $G$ has an $O T I S(p, q)$-layout if and only if there exist $p$ and $q$ with $p q=m$ and an isomorphism from $G=$ $(V, E)$ onto $H(p, q, d)$. We remark that if $G$ admits an $\operatorname{OTIS}(p, q)$-layout then $G^{-}$has an $\operatorname{OTIS}(q, p)$-layout, where $G^{-}$is the digraph obtained by reversing all the arcs of $G$.

We recall that it has been proved that the Imase and Itoh digraph, $I I(d, n)$, with $n$ nodes of degree $d$, has an $O T I S(d, n)$-layout [14], and consequently, so do the de Bruijn and the Kautz by Proposition 3.3. For fixed degree $d$, such a layout uses $p+q=O(n)$ lenses to interconnect $n$ processors, which is not very efficient. In the following we will show how to build an OTIS $(p, q)$ layout for the de Bruijn, such that the number of lenses used is $p+q=\Theta(\sqrt{n})$, which is also very efficient for practical values. We remark that the values of $p$ and $q$ determine also the size of the lenses and, by technological considerations, it is preferable to work with lenses of almost same size (i.e., $p \sim q$ ).

\subsection{The degree-diameter problem for OTIS lay- outs}

For given degree $d$ and diameter $D$, one can study the maximum number of nodes of the family of digraphs $H(p, q, d)$ (i.e., the digraph of degree $d$ built from an $O T I S(p, q))$. We obtained, by exhaustive search, the results reported in Table 1 for degree 2 and diameters 8 , 9 , and 10 . The table contains only the largest digraphs found for each diameter.

The Kautz digraph appears to be the largest digraph of degree $d$ and diameter $D$ which has an OTIS $(p, q)$ layout. This was predictable because it is among the best known constructions for the classic degree-diameter problem [8].

For fixed degree $d$, diameter $D$, and number of nodes $n=d^{D}$, we found several digraphs $H(p, q, d)$, with $p q=d^{D+1}$ for different values of $p$ and $q$ such that both $p$ and $q$ were powers of $d$. All these digraphs happened to be isomorphic to $B(d, D)$. As an example, $H(2,256,2), H(4,128,2)$ and $H(16,32,2)$ are isomorphic to $B(2,8)$. In the next section, we will prove that whenever $D$ is even and $H(p, q, d)$, as defined above, is connected, then it is isomorphic to $B(d, D)$. We will also address the case where $D$ is odd.

\subsection{Efficient $O T I S(p, q)$-layout of de Bruijn di- graphs}

In this section we characterize $\operatorname{OTIS}(p, q)$-layouts of de Bruijn digraph having a minimal number of lenses. For this, we will study cases where $p=d^{p^{\prime}}$ and $q=d^{q^{\prime}}$, as indicated in the discution above.

Proposition 4.1 The digraph $H(p, q, d)$ is isomorphic to $A\left(f, \mathcal{C}, p^{\prime}-1\right)$, where $\mathcal{C}$ is the complement permutation as defined in Definition 2.1, and where the permutation $f$ is defined on $Z_{D}, D=p^{\prime}+q^{\prime}-1$, as follows:

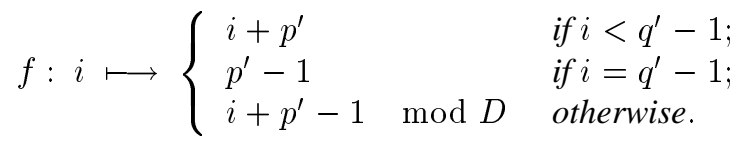




\begin{tabular}{|c|c|c|}
\hline $\mathrm{n}$ & $\mathrm{p}$ & $\mathrm{q}$ \\
\hline$\vdots$ & $\vdots$ & $\vdots$ \\
\hline 253 & 2 & 253 \\
\hline 254 & 2 & 254 \\
\hline 255 & 2 & 255 \\
\hline 256 & 2 & 256 \\
\cline { 2 - 3 } & 4 & 128 \\
\cline { 2 - 3 } & 16 & 32 \\
\hline 258 & 2 & 258 \\
\hline 264 & 2 & 264 \\
\hline 288 & 2 & 288 \\
\hline 384 & 2 & 384 \\
\hline \multicolumn{3}{|c|}{$D=8$} \\
\hline
\end{tabular}

\begin{tabular}{|c|c|c|c|}
\hline $\mathrm{n}$ & $\mathrm{p}$ & $\mathrm{q}$ \\
\hline$\vdots$ & $\vdots$ & $\vdots$ \\
\hline 509 & 2 & 509 \\
\hline 510 & 2 & 510 \\
\hline \multirow{4}{*}{$\mathrm{B}(2,8)$} & 511 & 2 & 511 \\
\cline { 2 - 4 } & 512 & 2 & 512 \\
\cline { 2 - 4 } & 8 & 128 \\
\hline 513 & 2 & 513 \\
\hline 516 & 2 & 516 \\
\hline & 528 & 2 & 528 \\
\hline & 576 & 2 & 576 \\
\cline { 2 - 4 } $\mathrm{K}(2,8)$ & 768 & 2 & 768 \\
\cline { 2 - 4 } & \multicolumn{3}{|c}{$D=9$} \\
& \multicolumn{3}{|c}{$D$}
\end{tabular}

$\mathrm{K}(2,9)$

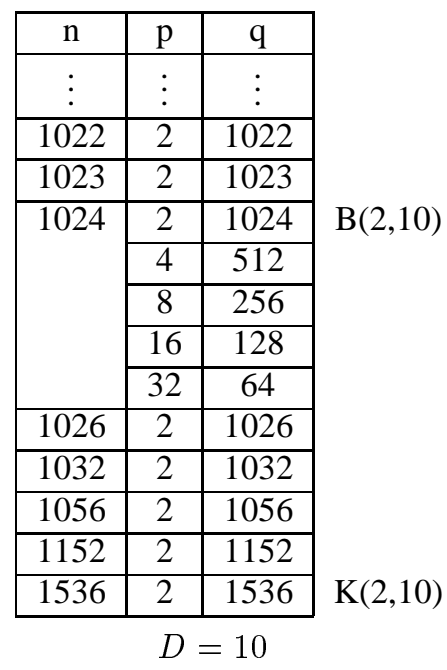

Table 1. $H(p, q, 2)$ with diameter $\mathbf{8 , 9}$ and $\mathbf{1 0 .}$
Proof: Notice that in this proof, we associate to a number $x \in Z_{d^{k}}$, its $d$-ary representation, denoted $\omega(x)$ (i.e., $\omega(x)=x_{k-1} \ldots x_{1} x_{0}, x_{i} \in Z_{d}$, such that $x=$ $\left.\sum_{i=0}^{k-1} x_{i} d^{i}\right)$.

Recall that the OTIS $(p, q)$ architecture connects $p$ groups of $q$ transmitters to $q$ groups of $p$ receivers, such that the transmitter $(i, j), 0 \leq i<p, 0 \leq j<q$, is connected to the receiver $(q-j-1, p-i-1)$. Remark that $\omega(i)$ has length $p^{\prime}$ and $\omega(j)$ has length $q^{\prime}$. Then, we label transceiver $(i, j)$ by $\omega(i) \omega(j)$, of length $p^{\prime}+q^{\prime}$. As $\omega(q-j-1)=\overline{\omega(j)}$ and $\omega(p-i-1)=\overline{\omega(i)}$, we have that the transmitter $\omega(i) \omega(j)$ is connected to the receiver $\overline{\omega(j) \omega(i)}$.

$H\left(d^{p^{\prime}}, d^{q^{\prime}}, d\right)$ has constant degree $d$ and $n$ nodes, and note that since $d$ divides both $p$ and $q$, each group of $d^{q^{\prime}}$ transmitters contains $d^{q^{\prime}-1}$ nodes. So we can label the $k$-th node $\left(k \in Z_{d^{q^{\prime}-1}}\right.$ ) of the $i$-th group of transmitters $\left(i \in Z_{p}\right)$ by $\omega(i) \omega(k)$, of length $p^{\prime}+q^{\prime}-1$. This node has the transmitters $\left\{(i, d k+\beta), \beta \in Z_{d}\right\}$, which are labeled by the set of strings $\left\{\omega(i) \omega(k) \omega(\beta), \beta \in Z_{d}\right\}$. Thus, the transmitters of the node $\omega(i) \omega(k)$ is connected to the receivers $\left\{\overline{\omega(k) \omega(\beta) \omega(i)}, \beta \in Z_{d}\right\}$. Let $\omega(i)=\omega(l) \omega(\alpha)$, where $\omega(l)$ is a string of length $p^{\prime}-1$ and $\alpha \in Z_{d}$. We have $\left\{\overline{\omega(k) \omega(\beta) \omega(i)}, \beta \in Z_{d}\right\}=$ $\left\{\overline{\omega(k) \omega(\beta) \omega(l) \omega(\alpha)}, \beta \in Z_{d}\right\}$ and these receivers belong to the nodes $\left\{\overline{\omega(k) \omega(\beta) \omega(l)}, \beta \in Z_{d}\right\}$.

Finally, a node $x$ represented by the string $\omega(x)=$ $\omega(l) \omega(\alpha) \omega(k)$, with $\omega(l) \in Z_{d}^{p^{\prime}-1}, \omega(\alpha) \in Z_{d}$ and $\omega(k) \in Z_{d}^{q^{\prime}-1}$, is connected through the $\operatorname{OTIS}(p, q)$ architecture, to the nodes represented by the strings $\left\{\overline{\omega(k) \omega(\beta) \omega(l)}, \beta \in Z_{d}\right\}$. As $\omega(x)=x_{D-1} \ldots x_{1} x_{0}$, $x_{i} \in Z_{d}$, we have $\omega(l)=x_{D-1} x_{D-2} \ldots x_{q^{\prime}}, \omega(\alpha)=$ $x_{q^{\prime}-1}$, and $\omega(k)=x_{q^{\prime}-2} \ldots x_{1} x_{0}$. Thus, the digraph $H\left(d^{p^{\prime}}, d^{q^{\prime}}, d\right)$ can be defined on the vertex set $Z_{d}^{D}$ with

$$
\Gamma_{H}^{+}\left(x_{D-1} \ldots x_{1} x_{0}\right)=\left\{\overline{x_{q^{\prime}-2} \ldots x_{0} \beta x_{D-1} \ldots x_{q^{\prime}}}\right\} .
$$

According to the definition of $f$ this is exactly $A\left(f, \mathcal{C}, p^{\prime}-1\right)$.

Corollary 4.2 Let $p^{\prime}+q^{\prime}-1=D$. For any degree $d$, the digraphs $B(d, D)$ and $H\left(d^{p^{\prime}}, d^{q^{\prime}}, d\right)$ are isomorphic if and only if the permutation $f$ of $Z_{D}$ defined by

$$
f: i \longmapsto \begin{cases}i+p^{\prime} & \text { if } i<q^{\prime}-1 ; \\ p^{\prime}-1 & \text { if } i=q^{\prime}-1 ; \\ i+p^{\prime}-1 \quad \bmod D & \text { otherwise }\end{cases}
$$

is cyclic.

Proof: We showed in Proposition 4.1 that $H\left(d^{p^{\prime}}, d^{q^{\prime}}, d\right)$ is isomorphic to the alphabet digraph $A\left(f, \mathcal{C}, p^{\prime}-1\right)$. We have also showed in Proposition 
3.9 that the alphabet digraph $A(f, \mathcal{C}, s)$ of degree $d$ and dimension $D$ is isomorphic to the de Bruijn digraph $B(d, D)$ when $f$ is cyclic. The corollary follows.

Now, we show that there exist $p^{\prime}$ and $q^{\prime}$ such that $B(d, D)$ and $H\left(d^{p^{\prime}}, d^{q^{\prime}}, d\right)$ are isomorphic and $d^{p^{\prime}}+$ $d^{q^{\prime}}=\Theta\left(\sqrt{d^{D}}\right)$ (i.e., $p+q=\Theta(\sqrt{n})$.

Proposition 4.3 Let $D$ be odd and $p^{\prime}=q^{\prime}=\frac{D+1}{2}$. Then $B(d, D)$ and $H\left(d^{\frac{D+1}{2}}, d^{\frac{D+1}{2}}, d\right)$ are isomorphic if and only if $D=1$.

Proof: If $p^{\prime}=q^{\prime}>1, f\left(p^{\prime}-1\right)=q^{\prime}-1=p^{\prime}-1$ and $f$ is not cyclic. If $p^{\prime}=q^{\prime}=1, D=1$ and $B(d, 1)$ is isomorphic to $H(d, d, d)$.

Corollary 4.4 Let $p^{\prime}=\frac{D}{2}$ and $q^{\prime}=\frac{D}{2}+1$. For fixed a degree $d$, if the diameter $D$ is even, the digraphs $B(d, D)$ and $H\left(d^{\frac{D}{2}}, d^{\frac{D}{2}+1}, d\right)$ are isomorphic, and therefore, $B(d, D)$ has an OTIS $(p, q)$-layout with $p+q=\Theta(\sqrt{n})$ lenses.

Proof: The permutation $f$ on $Z_{D}$ is defined by

$$
f: i \longmapsto \begin{cases}i+\frac{D}{2} & \text { if } i<\frac{D}{2} ; \\ \frac{D}{2}-1 & \text { if } i=\frac{D}{2} ; \\ i+\frac{D}{2}-1 \quad \bmod D & \text { otherwise }\end{cases}
$$

and one can easily verify that $f$ is cyclic. Thus, using Corollary 4.2 , the isomorphism is verified.

Figure 7 represents the digraph $H(4,8,2)$ with vertex set $Z_{2}^{4}$ and such that $\Gamma_{H}^{+}\left(x_{3} x_{2} x_{1} x_{0}\right)=\left\{\overline{x_{1} x_{0} \beta x_{3}}\right\}$, and Figure 8 represents the digraph $B(2,4)$ with the nodes labeling of $H(4,8,2)$.

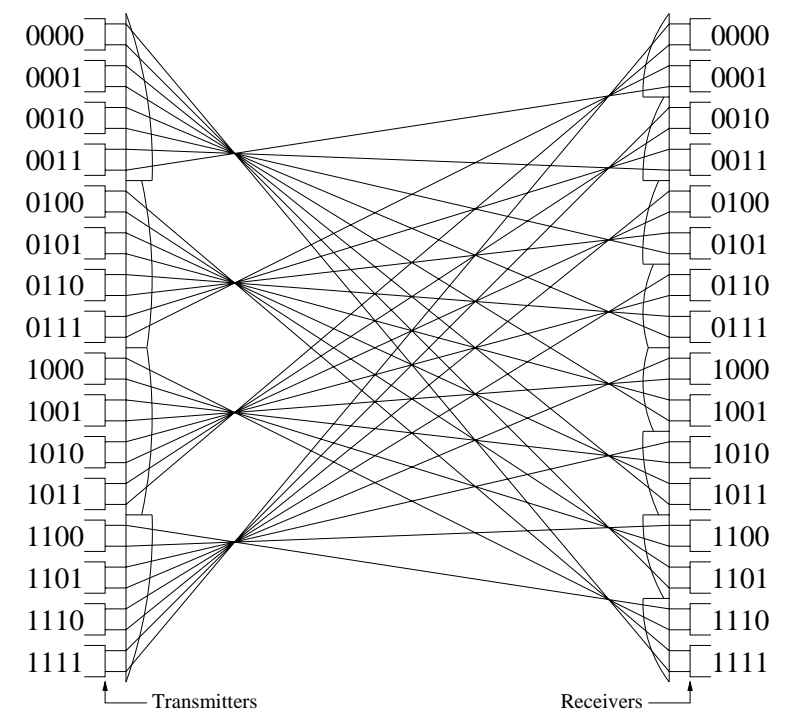

Figure 7. $H(4,8,2)$.

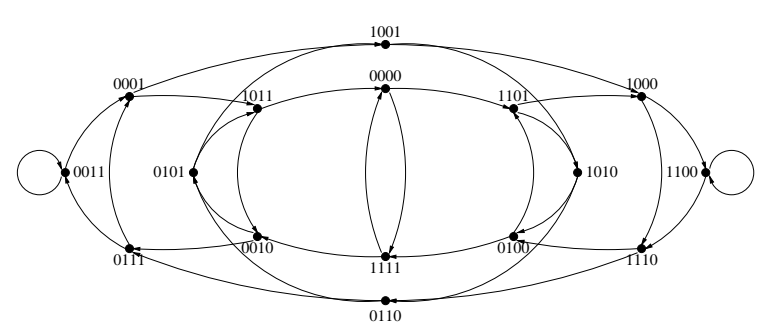

Figure 8. $B(2,4)$ with the adjacency relation of $H(4,8,2)$, i.e.: $\Gamma_{H}^{+}\left(x_{3} x_{2} x_{1} x_{0}\right)=$ $\left\{\overline{x_{1} x_{0} \beta x_{3}}\right\}$.

When the diameter $D>1$ is odd, by Proposition 4.3, $B(d, D)$ and $H(p, q, d)$ cannot be isomorphic with $p=q$. The case where $p$ is closest to $q$ is hence when, w.l.o.g., $p^{\prime}=\frac{D-1}{2}$ and $q^{\prime}=\frac{D-1}{2}+2$. However, it is not always the case that $B(d, D)$ and $H\left(d^{\frac{D-1}{2}}, d^{\frac{D-1}{2}+2}, d\right)$ are isomorphic. For instance, $H\left(2^{5}, 2^{7}, 2\right)$ and $B(2,11)$ are isomorphic, while $H\left(d^{6}, d^{8}, d\right)$ and $B(d, 13)$ are not.

Corollary 4.5 Given $B(d, D)$ and $H(p, q, d)$, their isomorphism can be verified in time $O(D)$.

Proof: Using Corollary 4.2 it is sufficient to verify that $f$ is a cyclic permutation of $Z_{D}$. This requires $O(D)$ steps.

Corollary 4.6 Minimizing $d^{p^{\prime}}+d^{q^{\prime}}$, such that $B(d, D)$ and $H\left(d^{p^{\prime}}, d^{q^{\prime}}, d\right)$ are isomorphic, requires $O\left(D^{2}\right)$ time steps.

Proof: If $D$ is even, using Corollary 4.4, the minimum is obtained with $p^{\prime}=\frac{D}{2}$ and $q^{\prime}=\frac{D}{2}+1$. Otherwise, there are $D$ pairs $\left(p^{\prime}, q^{\prime}\right)$, with $p^{\prime}<q^{\prime}$, such that $D=$ $p^{\prime}+q^{\prime}-1$. Thus, using Corollary 4.5, we obtain the minimum value in time $O\left(D^{2}\right)$.

\section{Conclusion}

In this paper, using graph theoretical properties that we proved, we characterized the $O T I S(p, q)$-layout of the de Bruijn digraph $B(d, D)$ when $p$ and $q$ are powers of $d\left(p=d^{p^{\prime}}, q=d^{q^{\prime}}\right.$ and $\left.p^{\prime}+q^{\prime}-1=D\right)$. Consequently, in the case where $d$ is prime, this implies a characterization of the OTIS $(p, q)$-layout of the de Bruijn. For the other cases, one could consider $\operatorname{OTIS}(p, q)$ layouts with $p, q \neq d^{i}$, but intuition and exhaustive search make us conjecture that, except for trivial cases, such layouts do not exist. 


\section{References}

[1] C. Berge. Graphes. Bordas, 1983.

[2] J-C. Bermond, R. Dawes, and F. Ergincan. De Bruijn and Kautz bus networks. Networks, 30:205218, 1997.

[3] J-C Bermond and P. Fraigniaud. Broadcasting and Gossiping in de Bruijn Networks. SIAM Journal on Computing, 23(1):212-225, 1994.

[4] J-C. Bermond, H.A Harutyunyan, A. Liestman, and S. Perennes. A note on the dimensionality of modified Knödel graphs. International Journal on Fondations of Computer Science, Special Issue on Interconnection Networks, 8(2):109-116, 1997.

[5] D. Van Blerkom, C. Fan, M. Blume, and S. Esener. Transimpedance receiver design optimization for smart pixel arrays. IEEE Journal of Lightwave Technology, 16(1):119-126, January 1998.

[6] M. Blume, G. Marsen, P. Marchand, and S. Esener. Optical Transpose Interconnection System for Vertical Emitters. OSA Topical Meeting on Optics in Computing, Lake Tahoe, March 1997.

[7] M. Blume, F. McCormick, P. Marchand, and S. Esener. Array interconnect systems based on lenslets and CGH. Technical Report 2537-22, SPIE International Symposium on Optical Science, Engineering and Instrumentation, San Diego (USA), 1995.

[8] W. Bridges and S. Toueg. On the impossibility of directed Moore graphs. Journal of Combinatorial Theory, B(29):339-341, 1980.

[9] F. Cao and A. Borchers. Optimal transmission schedules for lightwave networks embedded with de Bruijn graphs. Theoretical Computer Science, 222(1-2):113-131, 1999.

[10] D. Chiarulli, S. Levitan, R. Melhem, J. Teza, and G. Gravenstreter. Partitioned Optical Passive Star (POPS) Topologies for Multiprocessor Interconnection Networks with Distributed Control. IEEE Journal of Lightwave Technology, 14(7):16011612, 1996.

[11] O. Collins, S. Dolinar, R. McEliece, and F. Pollara. A VLSI decomposition of the de Bruijn graphs. Journal of the ACM, 39:931-949, 1992.

[12] C. Cooley and J. Tuckey. An algorithm for the machine calculation of complex Fourier series. Mathematics of Computation, 19:297-301, 1995.
[13] D. Coudert, A. Ferreira, and X. Muñoz. Multiprocessor Architectures Using Multi-hops MultiOPS Lightwave Networks and Distributed Control. In IEEE International Parallel Processing Symposium, pages 151-155. IEEE Press, 1998.

[14] D. Coudert, A. Ferreira, and X. Muñoz. OTISbased Multi-Hop Multi-OPS Lightwave Networks. In IEEE Workshop on Optics and Computer Science, number 1586 in Lecture Notes in Computer Science, pages 897-910. Springer verlag, 1999.

[15] P. Dapkus, M. MacDougal, G. Yang, and Y. Cheng. Ultralow threshold VCSELs for application to smart pixels. In Smart Pixels Technical Digest, page 5, Keystone, Colorado, August 5-9 1996. IEEE/LEOS Summer Topical Meetings.

[16] M. Feldman, S. Esener, C. Guest, and S. Lee. Comparison between electrical and free-space optical interconnects based on power and speed considerations. Applied Optics, 27(9):1742-1751, May 1988.

[17] D. Gardner, P. Marchand, P. Harvey, L. Hendrick, and S. Esener. Photorefractive Beamsplitter For Free Space Optical Interconnection Systems. OSA Applied Optics, 37(26):6178-6181, September 1998 .

[18] M. Ghisoni, H. Martinsson, N. Eriksson, M. Li, A. Larsson, J. Bengtsson, A. Khan, and G. Parry. 4x4 Fan-out Spot Generator Using GaAs Based VCSELs and Diffractive Optical Element. IEEE Photonics Technology Letters, 9:508, 1997.

[19] D. F. Hsu and D. S. L. Wei. Efficient Routing and Sorting Schemes for de Bruijn Networks. IEEE Transactions on Parallel and Distributed Systems, 8(11):1157-1170, 1997.

[20] M. Imase and M. Itoh. Design to Minimize Diameter on Building-Block Network. IEEE Transactions on Computers, 30(6):439-442, June 1981.

[21] M. Imase and M. Itoh. A Design for Directed Graphs with Minimum Diameter. IEEE Transactions on Computers, 32(8):782-784, August 1983.

[22] A. Jaekel, S. Bandyopadhyay, and A. Sengupta. A New Architecture for Multihop Optical Networks. In IEEE Workshop on Optics and Computer Science, number 1586 in Lecture Notes in Computer Science, pages 938-949. Springer verlag, 1999.

[23] W.H. Kautz. Bounds on directed (d,k) graphs. Theory of cellular logic networks and machines. AFCRL-68-0668 Final report, 20-28, 1968. 
[24] P. Marchand, F. Zane, R. Paturi, and S. Esener. Parallel Optoelectronic FFT Engine: Comparison to Electronic Implementations. submitted to Applied Optics, April 1997.

[25] G. Marsden, P. Marchand, P. Harvey, and S. Esener. Optical transpose interconnection system architectures. OSA Optics Letters, 18(13):10831085, July 1993.

[26] MEMS Optical Inc. http://www.memsoptical.com/.

[27] B. Mukherjee. Optical Communication Networks. Series on Computer Communications. McGrawHill, 1997.

[28] S. Perennes. Broadcasting and Gossiping on de Bruijn, Shuffle-Exchange and Similar Networks. DAMATH: Discrete Applied Mathematics and Combinatorial Operations Research and Computer Science, 83, 1998.

[29] S. M. Reddy, P. Raghavan, and J. G. Kuhl. A Class of Graphs for Processor Interconnection. In International Conference on Parallel Processing, pages 154-157. IEEE Computer Society Press, 1983.

[30] J. Rumeur. Communications dans les réseaux de processeurs. Masson, 1994.

[31] F. Sugihwo, M. Larson, and J. Harris. Low Threshold Continuously Tunable Vertical-CavitySurface-Emitting-Lasers with $19.1 \mathrm{~nm}$ Wavelength Range. Applied Physics Letters, 70:547, 1997.

[32] M. Syska. Communications dans les architectures à mémoire distribuée. $\mathrm{PhD}$ thesis, Université de Nice - Sophia Antipolis, 1992. http://www.essi.fr/ syska/biblio.html.

[33] G. Yayla, P. Marchand, and S. Esener. Energy Requirement and Speed Analysis of Electrical and Free-Space Optical Digital Interconnections. In P.Berthomé and A. Ferreira, editors, Optical Interconnections and Parallel Processing: Trends at the Interface, pages 49-128. Kluwer Academic, 1997.

[34] F. Zane, P. Marchand, R. Paturi, and S. Esener. Scalable Network Architectures Using The Optical Transpose Interconnection System. In Massively Parallel Processing using Optical Interconnections, pages 114-121, Maui, Hawaii, October 1996. IEEE Computer Society. 